\title{
Captures
}

Figures, théories et pratiques de l'imaginaire

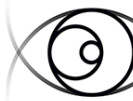

C A P T U R E S Figures, théories et pratiques de limagginaire evve interdisciplinaire

\section{Le temps presse}

\section{Les contrechants d'une histoire environnementale du Canada}

\section{Virginie Maris}

Volume 2, Number 1, 2017

Écrivains à l'écran

URI: https://id.erudit.org/iderudit/1059806ar

DOI: https://doi.org/10.7202/1059806ar

See table of contents

Publisher(s)

Figura, Centre de recherche sur le texte et l'imaginaire

ISSN

2371-1930 (digital)

Explore this journal

Cite this article

Maris, V. (2017). Le temps presse : les contrechants d'une histoire

environnementale du Canada. Captures, 2(1). https://doi.org/10.7202/1059806ar

\section{Article abstract}

L'exposition Le temps presse\&lt; présentée au Centre canadien d'architecture lors du 150e anniversaire du Canada propose une « contre-histoire environnementale du Canada ». J'expose ce récit et la façon dont il contribue à une critique nécessaire de la modernité. L'exposition participe davantage de l'autocritique d'un discours dominant centré sur lui-même qu'à une critique fondamentale du caractère colonialiste, patriarcal et anthropocentriste de la société contemporaine.
This document is protected by copyright law. Use of the services of Erudit (including reproduction) is subject to its terms and conditions, which can be viewed online.

https://apropos.erudit.org/en/users/policy-on-use/ 
Version enrichie de cet article : http://revuecaptures.org/node/753

\section{Le temps presse}

\section{Les contrechants d'une histoire environnementale du Canada}

\section{Virginie Maris}

Résumé :

L'exposition Le temps presse présentée au Centre canadien d'architecture lors du $150^{\circ}$ anniversaire du Canada propose une « contre-histoire environnementale du Canada ». J'expose ce récit et la façon dont il contribue à une critique nécessaire de la modernité. L'exposition participe davantage de l'autocritique d'un discours dominant centré sur lui-même qu'à une critique fondamentale du caractère colonialiste, patriarcal et anthropocentriste de la société contemporaine.

II y a presque un demi-siècle que l'on parle de crise environnementale. Dès les années 60 se sont fait entendre les échos des premiers lanceurs d'alertes. II s'agissait alors de documenter l'effet délétère des pesticides sur la biodiversité, comme dans le vibrant Silent Spring de Rachel Carson, qui dénonce les ravages du DDT sur les oiseaux communs et sur la santé humaine (Carson 1962); les risques d'explosion démographique pour les milieux naturels (Ehrlich, 1968); le déclin de la biodiversité (Ehrenfeld, 1970); le saccage des forêts tropicales (Myers, 1979); et plus généralement l'incompatibilité entre un modèle de croissance économique infinie et les limites biophysiques de la planète (Meadows, 1972). Progressivement, ces discours engagés ont atteint les institutions : ils se sont étayés d'une autorité scientifique toujours plus incontestable et ils ont été, en partie au moins, internalisés dans les politiques publiques.

Du côté des scientifiques, la crise est devenue un fait qu'il s'agit de documenter. Les taxinomistes tentent de recenser les espèces qui peuplent la terre plus vite que celles-ci ne s'éteignent, les statigraphes délibèrent sur le bien-fondé d'une nouvelle époque géologique caractérisée par l'influence des activités humaines sur le système planétaire, l'anthropocène (Waters, 2016). De nouvelles disciplines voient le jour, comme les sciences du système Terre, qui allient géologie, climatologie et écologie pour étudier les changements en cours, évaluant notamment les limites biophysiques de la planète et la mesure dans laquelle plusieurs d'entre elles ont déjà été dépassées (Rockström, 2009).

Du côté des politiques publiques, même si c'est avec des résultats encore bien insatisfaisants, la communauté internationale s'est engagée dans la lutte contre la désertification (CNULD, 1992), l'érosion de la biodiversité (CDB, 1992) ou encore les changements climatiques (CCNUCC, 1992). Les états se sont dotés d'agences et de ministères de l'environnement. Ils adoptent et révisent des stratégies nationales pour la conservation de la 
biodiversité, pour la qualité de l'air, pour la protection des grands fonds marins, etc. Mais ces formes d'institutionnalisation de l'action environnementale, si elles permettent peut-être de repousser la catastrophe, sont largement insuffisantes et ne semblent même pas suffire à ralentir le rythme de destruction des milieux naturels, de disparition des espèces, d'émissions de gaz à effet de serre.

En effet, ni l'avancement des connaissances ni les timides politiques publiques environnementales ne semblent à la hauteur de l'enjeu auquel se trouve aujourd'hui confrontée l'humanité : changer de façon significative les trajectoires de développement des sociétés modernes afin de les dévier de la catastrophe vers laquelle elles se dirigent. Seule une profonde transformation sociale serait susceptible de relever un tel défi. II s'agit d'inventer de nouvelles façons de vivre, d'habiter, de s'alimenter, de se représenter le monde. Pour cela, la philosophie, l'art, la littérature, la pédagogie, l'architecture sont tout aussi indispensables que la climatologie ou le Programme des Nations Unies pour l'environnement (PNUE). La question écologique se déploie aujourd'hui dans toutes les sphères de l'activité humaine, et il n'est donc pas surprenant que l'architecture, qui interroge directement notre façon d'habiter le monde, soit interpellée par la crise environnementale.

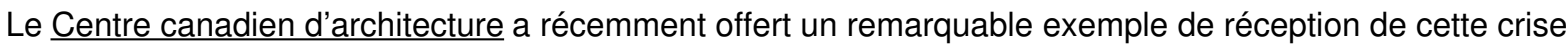
dans le champ de l'architecture et de l'aménagement du territoire, à travers une exposition intitulée « Le temps presse. Une contre-histoire environnementale du Canada moderne 1 ». Cette superbe exposition pose un regard critique sur le rapport complexe que les canadiens entretiennent avec leur environnement à travers un choix d'œuvres, de plans d'architecture et d'urbanisme, de documents de presse et d'installations artistiques. II y aurait beaucoup à dire sur la dimension muséographique, artistique et esthétique de ce travail, ainsi que sur l'audace politique d'un tel projet, mais je m'intéresserai principalement dans cet article à la trame philosophique sur laquelle se tisse ce récit. Plutôt que d'offrir une analyse ou une recension de l'exposition, je l'utilise comme prétexte à une autre balade, et j'invite le lecteur à me suivre sur les chemins de traverse, à explorer quelques sentiers discrets, à fureter dans les interstices de cette contre-histoire non pas tant pour critiquer ce qu'elle dit que pour dévoiler ce qu'elle ne dit pas. En soulignant les angles-morts de ce récit, je montrerai qu'il participe de l'autocritique salutaire d'un discours dominant sur lui-même mais qu'il tend néanmoins à omettre ou à minimiser les voix de véritables dissidences, voix dont l'écho permettrait d'envisager la crise environnementale contemporaine comme une facette d'un problème plus large de la modernité. Tout particulièrement significative à cet égard est la faible représentation de trois points de vue sur la crise environnementale contemporaine : celui des autochtones, celui des femmes et celui de la nature.

\section{Un exemple de réception : une exposition du Centre canadien d'architecture}

À l'occasion du $150^{\mathrm{e}}$ anniversaire du pays, le Centre canadien d'architecture (CCA) a présenté une exposition qui montre la façon dont l'architecture, l'urbanisme et l'aménagement du paysage au Canada sont interpellés 
par la crise environnementale contemporaine. Cette exposition formule une brillante démonstration de l'impasse dans laquelle la pensée moderne et son extension contemporaine dans la société de croissance et de consommation de masse nous ont menés. À travers le contexte particulier du Canada, et sous l'angle plus spécifique encore des enjeux d'architecture et d'aménagement du territoire, c'est une vaste réflexion critique sur la conception de la nature dans la culture occidentale qui s'amorce.

L'imaginaire canadien est en effet exemplaire du rapport paradoxal de la modernité à la nature : d'une part une « nature-sublime », vierge, idéalisée, comme celle d'un grand nord irréductiblement sauvage ou des forêts profondes et anciennes; d'autre part une «nature-utile », pourvoyeuse de ressources, qu'il convient d'assujettir, de gérer, matière inerte essentiellement disponible pour satisfaire nos besoins ou assouvir nos désirs, comme celle qui se dessinait dans la procession chaotique des billots d'épinettes le long de la rivière Saint-Maurice ou dans les mines de fer de Schefferville.

Dans l'exposition Le temps presse installée au CCA jusqu'au 9 avril 2017, le commissaire et directeur du Centre Mirko Zardini invite à découvrir ce qui se cache derrière les images d'Épinal de ces récits complémentaires : une histoire faite d'erreurs, de violence, d'accidents, de drames, l'histoire d'un saccage justifié par la nature-utile et camouflé par la nature-sublime.

Le chantier qu'il s'agit d'ouvrir part donc du besoin urgent de produire de nouveaux récits pour penser notre rapport à la nature. Mais pour inventer l'avenir encore faut-il savoir dire le passé, raconter cette histoire qui fonde et qui contraint le rapport des canadiens au territoire. Là se trouve donc le point de départ et l'ambition de l'exposition, dont le titre rappelle qu'il n'est plus possible de surseoir à cette tâche : produire une « contrehistoire environnementale du Canada moderne ", à rebours des deux grands récits du sublime et de l'utile; faire un état des lieux de ce rapport pathologique de la société canadienne à son environnement, et, à travers elle, peindre un portrait sans complaisance de la modernité occidentale.

Cette exposition nous propose de déambuler dans une succession de petites salles épurées qui mettent parfaitement en valeur le matériel documentaire choisi pour illustrer une série de catastrophes écologiques plus ou moins spectaculaires : coupures de presse, plans d'urbanisme, extraits audio ou vidéo, cartes, œuvres d'art, photographies, le tout accompagné de nombreux textes explicatifs. L'ensemble est remarquablement beau, lumineux, et la qualité esthétique de la forme permet d'apaiser un peu le sentiment anxiogène que génère le contenu. Le visiteur y parcourt cette (contre)histoire environnementale à travers le temps, l'histoire de l'extraction et de la surexploitation des ressources naturelles étant presque aussi vieille que celle de la colonisation elle-même, et à travers l'espace, la crise environnementale nous rappelant violemment notre dépendance à un territoire bien plus vaste que celui que nous occupons consciemment. C'est un voyage dans le temps grâce aux nombreux documents d'archive qui balisent l'exposition. Les utopies technophiles des 
années 50 sont restituées à travers une série de photos, de plans, de coupes géologiques qui retracent le développement nucléaire de Chalk River; les accords de la Baie-James sont évoqués dans des extraits de films et divers documents d'archives; les grandes crises de pollution et les scandales sanitaires qui ont ponctué le dernier demi-siècle s'égrènent au fil des coupures de presse et des micro-trottoirs des grandes chaînes d'information; la naissance et l'évolution des parcs nationaux sont évoquées dans la dernière salle...

C'est un voyage dans l'espace grâce à une multitude de formes visuelles habilement mises en valeur par une scénographie qui érige certains documents techniques en véritables œuvres d'art. Les cartes géographiques sont nombreuses, belles, explicites, elles nous font découvrir le monde vu du Nord et sa calotte glacière, qui d'année en année se réduit comme peau de chagrin; elles nous font visiter l'étendue infinie de la terre de Baffin, immense espace vierge pourtant source des plus grandes convoitises; elles nous dessinent des réseaux superposés de ressources naturelles, de populations, de transports, serrés dans le Sud pour faire voyager les humains sur la terre, plus lâches dans le Nord pour que circulent les ressources sous le sol.

C'est ainsi que l'on parcourt l'histoire et le territoire canadiens, jusqu'à ce point ultime où le temps et l'espace semblent ne faire plus qu'un dans le blanc suspendu du Grand Nord. C'est ce que donne à voir la série magnifique de Donovan Wylie, qui photographie les paysages lunaires de l'Arctique canadien, d'où saillent les stations radar de la ligne DEW, cette « ligne avancée d'alerte précoce » (Distant Early Warning Line), gigantesque réseau de radars construit dans les années 50 qui s'étendait de l'Alaska à l'Islande et devait prémunir l'Ouest d'une éventuelle attaque soviétique. Ces vestiges gigantesques, comme autant de cicatrices sur l'étendue immaculée de l'Arctique canadien que le développement des technologies d'observation par satellite a rapidement rendu obsolètes, témoignent de l'acharnement d'une civilisation guerrière à marquer et à défendre son territoire, quand bien même il lui faudrait pour cela commencer par le saccager.

\section{Quelles voix pour dire la catastrophe?}

Que ce soit pour dresser l'inventaire des échecs passés ou pour esquisser le récit d'un avenir meilleur, il faudrait donner à entendre d'autres voix que celles qui généralement saturent l'espace public. Une contrehistoire ici pourrait être conçue comme un contre-chant atonal, un chœur alternatif et discordant qui s'exprimerait dans un autre mode que celui des harmonies lisses et factices qu'a façonnées la modernité pour chanter ses propres louanges. Or la crise environnementale actuelle, que le jargon scientifico-technique renomme pudiquement « anthropocène », est celle de la triple domination colonialiste, patriarcale et anthropocentrique de l'homme blanc. C'est donc vers les sans-voix de cette suprématie qu'il faudrait tendre l'oreille : les peuples autochtones, les femmes et la nature elle-même ont probablement beaucoup à nous dire.

Or, si cette remarquable exposition relève avec succès le défi du mea culpa d'une culture occidentale acculée 
au constat de ses propres échecs, la contre-histoire qu'elle nous offre ne déploie pas complètement son projet de récit alternatif. Les voix dominantes restent les voix des dominants, et les visions du monde qui s'y exposent ne permettent pas d'ébranler les rapports d'asservissement constitutifs de la modernité et de la crise environnementale.

\section{La voix des autochtones - pour sortir du colonialisme}

L'exposition fait une part belle aux peuples autochtones : Premières Nations, Inuits, Métis sont à plusieurs reprises convoqués, essentiellement selon deux modalités. D'une part, l'exposition nous rappelle l'importance des luttes qui ont été menées pour la reconnaissance de leurs droits, que ce soit en termes de gestion territoriale, même si ce n'est que de façon rudimentaire et insatisfaisante comme lors des accords de la BaieJames; ou de pratiques traditionnelles comme dans l'affaire Donald Marshall en 1999, qui finit par reconnaître le droit issu des traités des Mi'kmaq et des Malécites à pêcher dans les Grands Bancs en dépit du moratoire sur la morue.

D'autre part, plusieurs situations d'injustice environnementale sont documentées dans l'exposition. De façon presque systématique, les coûts et les risques associés à la dégradation des milieux naturels et à la surexploitation des ressources pèsent plus lourd sur les épaules des populations qui sont déjà vulnérables, les inégalités environnementales venant s'accumuler en quelque sorte sur les injustices politiques, culturelles ou économiques déjà existantes. Et dans la grande distribution des bénéfices et des pouvoirs, les peuples autochtones sont probablement les communautés pour lesquelles la convergence entre les injustices sociopolitiques et environnementales est la plus forte : d'abord, parce que les valeurs culturelles qui lient ces communautés à leur milieu sont plus denses et plus complexes que dans la culture occidentale dominante (Westra, 2008); ensuite parce que les modes de vie autochtones sont plus étroitement dépendants de la nature, la pêche et la chasse occupant une place importante dans l'alimentation, l'économie et l'identité culturelle de nombreuses communautés; enfin, parce que les industries extractives et polluantes les plus dommageables à l'environnement sont souvent situées sur ou à proximité de territoires autochtones. C'est ainsi qu'on apprend avec stupeur que, dans le pays, en 2015, $20 \%$ des communautés autochtones faisaient l'objet d'un avis d'ébullition faute d'avoir accès à une eau potable sécuritaire, le triste record étant détenu par les résidents de la Première Nation Neskantaga au nord-ouest de l'Ontario, qui n'ont pas pu boire l'eau du robinet depuis 20 ans. En Alberta, sur les rives de la rivière Athabasca contaminée par des fuites de résidus des sables bitumineux, des impacts importants sur la santé des Premières Nations Chipewyan et Mikisew Cree sont connus, l'exposition aux métaux lourds et le développement de cancer étant anormalement élevés chez les résidents de ces communautés (Hoover, 2012).

Mais les voix autochtones ne sont pas seulement celles des luttes et des complaintes. Elles sont également 
porteuses de savoirs et d'espoir, elles ouvrent nos consciences à d'autres visions du monde, d'autres façons de cœxister avec les vivants non-humains, d'autres ontologies que le dualisme moderne qui sépare les humains de la nature (Descola, 2005). Dans cette contre-histoire de l'environnement que nous avons à écrire, il serait utile de se tourner aussi vers les contributions actives de ces communautés à l'invention d'un modèle de vie durable, qui ne soit ni folklorique ni passéiste mais qui prenne appui sur les longues traditions de coopération avec le monde vivant de ces sociétés bien plus durables que le modèle dominant (Berkes, 2012). Alors que l'exposition réussit dans une large mesure à se faire la porte-parole des revendications autochtones, elle peine à faire entendre ce que ces cultures ont à nous dire et à nous enseigner de leur façon d'habiter le monde.

\section{La voix des femmes - pour sortir du patriarcat}

Dans son ouvrage The Death of Nature, Carolyn Merchant (1990) analyse la façon dont les deux faces de l'iconographie occidentale de la nature reflètent les masques contraires dont la pensée chrétienne a recouvert le visage des femmes : la vierge, parfaite, immobile, intouchable; et la femme quotidienne qu'il revient à I'homme de domestiquer, de fertiliser, de rendre productive, une femme-ventre, nourricière, nulle part mieux à sa place que sur la table d'accouchement ou derrière les fourneaux. S'affranchir du vieux récit qui verrouille le champ des possibles, c'est donc aussi laisser aux femmes la possibilité de se raconter elles-mêmes, de s'inventer, de dévoiler sans peur le savoir ancestral des sorcières. Car si elles peuvent être mères, elles savent également comment ne pas enfanter; si elles nourrissent les leurs, elles peuvent aussi leur concocter des cures et des poisons. Ces savoirs prennent racines dans un rapport bien plus immédiat à la nature que celui d'une science moderne désincarnée qui voit dans la domination l'expression de sa force et dans le progrès technique la seule voie de son salut (Plumwood, 2001).

Plus encore que pour les voix autochtones, la trop discrète présence des femmes dans cette exposition est regrettable. Elle surgit dans la blancheur insouciante d'une robe qui tournoie en dansant une scottish que l'on observe confortablement installé sur un canapé vintage. L'immense photographie nous plonge dans le salon d'un petit pavillon de Deep River en 1958. La même année, à quelques kilomètres de là, des barres de combustibles prennent feu dans le réacteur NRU de Chalk River, contaminant l'eau, l'air, la forêt. La femme ici et dans plusieurs photographies de la même série ne sert qu'à illustrer la vie paisible et confortable qui se généralise alors mais dont l'innocence tranche avec le prix à payer : une longue succession d'accidents fuites, fusions, contaminations - , l'absence totale de perspectives durables pour le stockage des déchets, et l'ombre qui plane d'une catastrophe majeure comme celles qui dévastèrent Tchernobyl ou Fukushima semblent écartées par le geste féminin et gracieux de cette danse de salon, chacun rêvant confusément que le pire advienne suffisamment tard pour n'avoir pas à en être témoin. 
Le visage de la femme est furtivement convoqué dans un reportage télévisé, mère inquiète de la santé de ses enfants, infirmière débordée, institutrice en colère, dans la petite communauté de Walkertown en Ontario, dont le réseau d'eau potable s'est trouvé contaminé par une souche dangereuse d'Escherichia Coli en mai 2000, provoquant le décès de sept personnes et contaminant plus de 2000 habitants. Un orage, une agriculture intensive productrice de lisier, des déversements non contrôlés, une négligence devenue systémique à force de privatisations et de réductions des investissements publics, et voilà que le drame s'insinue dans le quotidien de ce petit village, s'infiltre dans les maisons et dans les corps, montrant soudain au pays tout entier comme nous sommes vulnérables et dépendants.

Une vidéo retransmet le témoignage d'une activiste de Greenpeace qui, avec deux autres grimpeurs, escalade en 1984 l'immense cheminée qui se dresse dans le ciel ontarien pour disséminer les particules mortelles que crache à des kilomètres à la ronde l'industrie minière. Et c'est à peu près tout. Une dizaine de voix féminines à peine dans l'interminable galerie d'hommes, politiciens, scientifiques, journalistes, militants, architectes, artistes, des hommes qui disent en hommes le monde de l'arrogance, de l'hubris, de la confiance coupable en leur propre pouvoir et de l'insatiable appétit de puissance et de possession. Alors sûrement faudrait-il, pour composer ce monde à venir, écouter la voix des femmes, celle qui fredonne et murmure, celle qui raconte, celle qui chante, celle qui se bat et se défend (Hache, 2016).

\section{La voix des non-humains - pour sortir de l'anthropocentrisme}

La dernière grande figure trop absente de cette contre-histoire est celle des êtres de nature, écosystèmes, animaux sauvages, formations géologiques, arbres, autant d'entités qui sont notre environnement au moins autant qu'elles le partagent avec nous, et dont il n'est finalement presque jamais question. Elle n'apparaît que furtivement, toujours dans les marges ou les détours de ce qui fait le centre du propos. Quelques ours traversent nonchalamment la transcanadienne par un passage faunique du parc Banff, à la sortie duquel un piège photographique capture également la silhouette majestueuse d'un cougar ou le regard inquiet d'un orignal. II y a ce caribou qui observe de loin le prototype d'une ville fortifiée d'Arctique dans un magnifique croquis de Ralph Erskine réalisé en 1958. II y a ces rangées de morues réduites à leur plus pure expression de ressources, visualisation froide et monochrome d'un stock en chute libre. Il y a cet ours polaire invisible mais redouté des ouvriers chargés du démantèlement de la ligne DEW et qui pourrait à tout instant surgir du brouillard. II y a la forêt, qu'on ne voit que d'en haut, trop distante pour y distinguer les essences, y deviner la faune, une forêt comme une plage verte sur une carte topographique, des bosquets improbables inventés par un logiciel de design dans le projet Tree City à Toronto.

Car dans le récit qu'il nous faudra produire pour inventer un nouveau rapport au monde, il conviendrait de penser avec la nature plutôt que de le faireà côté d'elle comme c'est souvent le cas dans cette exposition. 
Sortir justement de cette idée d'environnement qui conditionne l'exposition de l'histoire passée pour le réinventer comme un milieu, un partenaire, une communauté d'entités autonomes qu'il convient de reconnaître et avec lesquelles il est grand temps d'entrer en dialogue (Callicott, 1989), quitte à devoir pour cela inventer de nouveaux langages (Morizot, 2016).

II aurait été intéressant de voir se surimposer aux sinistres réseaux de gazoducs et de voies ferrées les corridors migratoires, les biomes, les aires de distribution des espèces qui partagent avec nous le territoire et I'habitent souvent d'une façon tellement plus délicate et performante que nous ne savons le faire. On aurait aimé s'interroger sur ce que cela peut être, le Canada, pour une grande oie des neiges qui chaque année le traverse dans un sens puis dans l'autre.

\section{Quelques sentiers tracés pour habiter autrement la Terre}

Plusieurs projets sont présentés dans l'exposition esquissant des alternatives, nous montrant que cela fait longtemps déjà que l'on imagine d'autres trajectoires, d'autres façons d'habiter la Terre : vivre dans le Grand Nord dans une cité arctique écologique, concevoir des habitats autonomes, capables de produire eux-mêmes l'énergie nécessaire à ses habitants mais aussi de pourvoir à leur besoins alimentaires, imaginer des campings confortables mais respectueux de la nature, etc. Ce qui apparaît dans la plupart de ces projets, c'est une inflexion du « faire contre » la nature vers un « faire avec ». Se servir des ressources, de l'énergie disponible, plier comme un roseau soufflé par le vent plutôt que de résister, inébranlable, jusqu'au déracinement fatal. Beaucoup de ces projets ne sont à ce jour rien d'autre que cela, des projets, des croquis, des plans, des esquisses, et l'on espère bien sûr qu'ils seront de plus en plus nombreux à devenir des réalités tangibles, des maisons, des rues, des villes intelligentes et décarbonnées. Néanmoins, lorsqu'on prend la mesure véritable du bilan que dresse cette exposition, et au-delà du constat de la crise sans précédent que traverse le monde vivant, si l'on admet qu'en deux siècles à peine le modèle productiviste occidental a détruit ou dégradé plus des deux tiers des milieux naturels terrestres (Millennium Ecosystem Assessment, 2005), que la moitié des espèces risque de disparaître avant la fin du siècle (Billé, 2014), alors il ne suffit plus de « faire avec ». Ce que l'on peut demander à l'urbanisme, à l'aménagement, à l'architecture aujourd'hui, c'est aussi de « faire pour ». Les quelques tunnels pour éviter les collisions avec la faune sauvage ne suffiront pas. II est temps de penser, dans notre façon d'habiter le monde, ce que l'on peut activement offrir à la nature, pour qu'entre chaque dalle poussent des herbes folles, que dans chaque anfractuosité nichent des coléoptères et des guêpes maçonnes, que nos toits servent d'abri aux hirondelles, que nos jardins, nos balcons, les bords de nos fenêtres deviennent le terrain de jeu des bruants, mésanges, et autres cistudes.

C'est à la toute fin de l'exposition que l'on trouve quelque chose comme une synthèse de ces trois voix dans l'admirable travail de « restauration invisible » de Cornelia Hahn Oberland. Cette expression traduit la notion 
d'invisible mending, qui désigne les opérations de stoppage consistant à reconstituer minutieusement à la fois la trame et la chaîne d'un tissu abîmé par un accroc, une brûlure ou un coup de ciseau accidentel. L'architecte paysagiste adopte une démarche analogue dans l'aménagement de l'Assemblée législative des Territoires du Nord-Ouest construite à Yellowknife en 1993. En amont des travaux de construction du bâtiment, le site est minutieusement échantillonné, une immense quantité de plantes vouées à la destruction sont collectées, transférées puis replantées une à une à l'issue des travaux, de telle sorte que le bâtiment pourtant très imposant se retrouve dans un écrin de végétation autochtone. Tout le talent et les efforts sont alors dédiés à ce que justement ni talent ni effort ne soient visibles. II n'est pas question ici de réparer la catastrophe, comme à Sudbury où c'est à coups de déversements chimiques qu'il a fallu désacidifier un sol que quelques décennies d'activité industrielle ont rendu complètement stérile, ni d'effacer à grands frais les stigmates d'un développement titanesque que le temps et les innovations ont rendu désuets comme dans le démantèlement de la ligne DEW, mais de penser en amont, humblement, patiemment, à l'effacement de nos traces. Le projet d'architecture paysagère de l'Assemblée législative des Territoires du Nord-Ouest nous convainc, juste avant que s'achève la visite, qu'il est possible de penser d'un même souffle les institutions de justice de toutes les communautés et le respect de la nature et des êtres vivants qui la peuplent.

À travers cette contre-histoire environnementale du Canada, c'est toute l'histoire d'une société qui se dessine, parfois en relief, une histoire de luttes, de pouvoirs, de négociations, parfois en creux, une histoire d'absence, de secrets, d'invisibilité. C'est en tout cas une occasion inestimable de s'interroger sur ce qui fait notre identité et sur ce que nous souhaitons construire ou déconstruire pour habiter le monde d'une façon plus respectueuse, plus juste et plus soutenable.

Parce que la crise doit nous inviter à réfléchir au système qui l'a produite, il ne faut pas laisser à ce système lui-même le soin de formuler et de résoudre les problèmes auxquels nous faisons face. II ne faut pas laisser les géants de l'industrie pétrolière chercher pour nous des solutions aux changements climatiques. II ne faut pas attendre que les multinationales agroindustrielles réduisent la faim dans le monde. II ne faut pas espérer que la finance mondiale règle les injustices économiques. Plus généralement, il n'est pas raisonnable de croire qu'une société de croissance fondée sur l'exploitation des peuples, des femmes et de la nature puisse, sans bouleversement majeur, se réformer pour suivre la voie radieuse d'un développement dit durable (Maris, 2006). Or, il en va de même pour la production des récits que pour la production des semences. Si le temps presse d'inventer de nouvelles formes pour raconter l'échec de la culture occidentale, alors il faut le faire avec d'autres voix, d'autres mots, d'autres chants que ceux qui ont façonné cette culture. L'autocritique est certainement salutaire et on peut l'envisager comme un premier pas utile et peut-être même nécessaire, ne serait-ce que pour convaincre les privilégiés de céder un peu du terrain. II convient néanmoins de veiller à ce que le mea culpa des dominants ne soit qu'un prélude à une multitude d'autres paroles et qu'il ne serve pas 
finalement à rendre plus durables et plus profondes les formes d'aliénation qui ont fondé leur hégémonie.

1. L'exposition_est maintenant à l'affiche à Toronto, au University of Toronto Art Centre, jusqu'au 15 juillet 2017. Le lecteur pourra également se reporter au superbe catalogue d'exposition qui a été produit pour accompagner l'événement (Bratishenko, 2016).

\section{Bibliographie}

Berkes, Fikret. 2012. Sacred Ecology, 3e édition. New York : Routledge, 392 p.

BııLé, Raphaël, Philippe Cury, Michel Loreau et Virginie MarIs. 2014. Biodiversité. Vers une sixième extinction de masse? Montreuil : La ville brûle, 224 p.

Bratishenko, Lev et Mirko ZardinI (dir.). 2016. Le temps presse. Une contre-histoire environnementale du Canada moderne, catalogue de l'exposition organisée et présentée au CCA du 16 novembre 2016 au 9 avril 2017. Montréal; Heijningen (Pays-Bas) : Centre canadien d'architecture; Jap Sam Books, 364 p.

CALLICOTT, Baird J. 1989. In Defense of the Land Ethics. Essays in Environmental Philosophy New York : State University of New York Press, 336 p.

Carson, Rachel. 1962. Silent Spring. Boston : Houghton Mifflin, 368 p.

Descola, Philippe. 2005. Par-delà nature et culture. Paris : Gallimard, 640 p.

Ehrenfeld, David W. 1970. Conserving life on earth. New York : Oxford University Press, 360 p.

Ehrich, Paul R. 1968. The Population Bomb. New York : Ballantine Books, 223 p.

HACHE, Émilie. 2016. Reclaim . Recueil de textes écoféministes. Paris : Cambourakis, 412 p.

Hoover, Elizabeth, Katsi Cook, Ron Plain, Kathy Sanchez et al.. 2012. «Indigenous Peoples of North America. Environmental Exposures and Reproductive Justice ». Environmental Health Perspectives, vol. 120 , no 12, p. 1645-1649.

MARIS, Virginie. 2006. «Le développement durable. Enfant prodigue ou rejeton matriphage de la protection de la nature? ». Les ateliers de l'éthique, vol. 1, no 2, 86-102.

Meadows, Donella H, Dennis L Meadows, Jorgen Randers et William W Behrens. 1972. Limits to Growth. A Report for The Club of Rome's Project on the Predicament of Mankind. New York : Univers Books, $205 \mathrm{p}$.

Merchant, Carolyn. 1990. The Death of Nature. Women, Ecology, and the Scientific Revolution reprint edition. New York : HarperOne, $384 \mathrm{p}$.

Assessment, Millenium Ecosystem. 2005. Ecosystem and Human Well-being. Synthesis Washington : Island 
Press, $137 \mathrm{p}$.

Morizot, Baptiste. 2016. Les Diplomates. Cohabiter avec les loups sur une nouvelle carte du vivant Paris : Wildproject Editions, $320 \mathrm{p}$.

Myers, Norman. 1979. The Sinking Ark. A New Look at the Problem of Disappearing Species Oxford :

Pergamon, $307 \mathrm{p}$.

Plumwood, Val. 2001. Environmental Culture. The Ecological Crisis of Reason New York : Routledge, $300 \mathrm{p}$.

Rockström, Johan, Will Steffen, Kevin Noone, Åsa Persson et al.. 2009. «Planetary Boundaries. Exploring the Safe Operating Space for Humanity ». Ecology and Society, vol. 14, no 2 « article 32 ».

Waters, Colin N, Jan Zalasiewicz, Colin Summerhayes, Anthony D Barnosky et al.. 2016. «The Anthropocene is functionally and stratigraphically distinct from the Holocene ». Science, vol. 351, no 6269. <http://science.sciencemag.org/content/351/6269/aad2622>. Consultée le 31 mai 2016.

WeStRA, Laura. 2012. Environmental Justice and the Rights of Indigenous Peoples. International and Domestic Legal Perspectives. Londres : Earthscan, 352 p. 